\title{
$1.9 \%$ bi-axial tensile strain in thick germanium suspended membranes fabricated in optical germanium-on-insulator substrates for laser applications
}

\author{
A. Gassenq, ${ }^{1,2}$ K. Guilloy, ${ }^{1,2}$ G. Osvaldo Dias, ${ }^{1,3}$ N. Pauc, ${ }^{1,2}$ D. Rouchon,,${ }^{1,3}$ \\ J.-M. Hartmann, ${ }^{1,3}$ J. Widiez, ${ }^{1,3}$ S. Tardif, ${ }^{1,2}$ F. Rieutord, ${ }^{1,2}$ J. Escalante, ${ }^{1,2}$ I. Duchemin ${ }^{1,2}$ \\ Y.-M. Niquet, ${ }^{1,2}$ R. Geiger, ${ }^{4}$ T. Zabel, ${ }^{4}$ H. Sigg, ${ }^{4}$ J. Faist ${ }^{5}$ A. Chelnokov, ${ }^{1,3}$ V. Reboud, ${ }^{1,3}$ \\ and V. Calvo ${ }^{1,2}$ \\ ${ }^{1}$ Univ. Grenoble Alpes, 38000 Grenoble, France \\ ${ }^{2}$ CEA-INAC, SP2M, 17 rue des Martyrs, 38000 Grenoble, France \\ ${ }^{3}$ CEA-LETI, Minatec Campus, 17 rue des Martyrs, 38054 Grenoble, France \\ ${ }^{4}$ Laboratory for Micro- and Nanotechnology, Paul Scherrer Institut, 5232 Villigen, Switzerland \\ ${ }^{5}$ Institute for Quantum Electronics, ETH Zurich, 8093 Zürich, Switzerland
}

(Received 3 August 2015; accepted 31 October 2015; published online 11 November 2015)

\begin{abstract}
High tensile strains in Ge are currently studied for the development of integrated laser sources on $\mathrm{Si}$. In this work, we developed specific Germanium-On-Insulator $200 \mathrm{~mm}$ wafer to improve tolerance to high strains induced via shaping of the Ge layers into micro-bridges. Building on the high crystalline quality, we demonstrate bi-axial tensile strain of $1.9 \%$, which is currently the highest reported value measured in thick $(350 \mathrm{~nm})$ Ge layer. Since this strain is generally considered as the onset of the direct bandgap in Ge, our realization paves the way towards mid-infrared lasers fully compatible with CMOS fab technology. (C) 2015 AIP Publishing LLC.
\end{abstract}

[http://dx.doi.org/10.1063/1.4935590]

Silicon $(\mathrm{Si})$ and Germanium $(\mathrm{Ge})$ are widely used as source/channel/drain materials in nano-electronics integrated circuits. However, the indirect bandgap of these group IV elements prevents them from being used as efficient lightemitters. Different approaches have been proposed to overcome the indirect bandgap of $\mathrm{Ge}$, such as doping, ${ }^{1}$ strain adding, ${ }^{2,3}$ or $\mathrm{Sn}$ alloying. ${ }^{4}$ According to calculations, between $1.7 \%$ and $1.9 \%$, biaxial tensile strain transforms Ge into a direct semiconductor with a direct bandgap in the midinfrared around $2.6 \mu \mathrm{m}$ wavelength. ${ }^{5-8}$ Concerning bi-axial strain, $44 \mathrm{~nm}$ thin Ge membranes glued on kapton membrane and deformed by a high pressure gas were reported to withstand a tensile strain of up to $2.0 \% .^{9}$ The highest bi-axial tensile strain reported so far, $2.35 \%$, was achieved in even thinner $(10 \mathrm{~nm})$ Ge layers epitaxially grown on larger lattice parameter InGaAs buffers. ${ }^{10}$ Such small thicknesses prevent them from being used to confine light in photonics devices because of a dramatically reduced overlap with the optical field. SiN stressor layers were also used to induce strain in $\mathrm{Ge},{ }^{11,12}$ but the achieved strain values were rather lower. We consider suspended Ge membranes as most interesting to reach the highest levels of strain, using strain redistribution. $^{2,3,6}$ The highly strained membranes are envisioned to be used as gain media inserted in a laser cavity defined, for instance, by photonics crystals. ${ }^{13}$

In this paper, we focus our work in reaching such challenging high level of strain predicted as an indirect-direct transition. We detail how we obtained a high bi-axial strain in thick Ge $(0.35 \mu \mathrm{m})$ by using strain redistribution in Ge micro-bridges. This was made possible, thanks to membrane processing in high quality optical GeOI substrates with a low density of threading dislocations. The thick Ge withstands high strains, while providing optical confinement and low propagation losses of the optical modes.
First, the $200 \mathrm{~mm}$ optical GeOI wafers fabrication using the Smart $\mathrm{Cut}^{\mathrm{TM}}$ technology will be explained. Then, the principle and the process flow used for micro-bridge fabrication will be detailed. Finally, the strain characterization will be presented.

The process flow starts with the "optical" GeOI substrate fabrication presented in Figure 1. GeOI substrates fabricated using the Smart $\mathrm{Cut}^{\mathrm{TM}}$ technology were initially developed for microelectronic purposes; Ge overlayers and Buried Oxide (BOX) were thus thin $(60$ and $150 \mathrm{~nm}$, typically). ${ }^{14,15}$ However, such thicknesses prevent them from being used for photonics, which requires thick Ge and BOX layers for light confinement and low leakage into the substrate. ${ }^{16}$ We developed specific processing steps to fabricate "optical" GeOI substrates. First, a $2.5 \mu \mathrm{m}$ intrinsic Ge layer was grown on $200 \mathrm{~mm} \mathrm{Si} \mathrm{wafers} \mathrm{in} \mathrm{an} \mathrm{Epi} \mathrm{Centura} \mathrm{reduced}$ pressure-chemical vapor deposition tool (Figure 1(b)). Short thermal cycling was used afterwards to reduce the threading dislocations density (down to $10^{7} \mathrm{~cm}^{-2}$ ). A $2 \mathrm{~nm}$ thick $\mathrm{Si}$ passivation layer was deposited on the $2.5 \mu$ m thick Ge layer to protect it from oxidation. A thin silicon di-oxide layer $(200 \mathrm{~nm})$ was then deposited by PECVD on top of the Ge layer (Figure 1(c)) followed by $\mathrm{H}^{+}$ion implantation (Figure $1(\mathrm{~d})$ ) in order to locally create defects and allow splitting in the Ge layer. A thick thermal $\mathrm{SiO}_{2}$ layer was grown on the $\mathrm{Si}$ handle substrate. Oxides on both substrates were polished prior to direct bonding (Figure 1(e)). The bonded pair splits during the subsequent thermal anneal (Figure 1(g)), yielding a GeOI substrate and liberating the donor wafer that can then be prepared for reuse. Surface roughness originating from the splitting step was eliminated by Chemical Mechanical Polishing (CMP) (Figure 1(h)). After CMP, the Ge film is roughly $1 \mu \mathrm{m}$ thick (on top of a $1 \mu \mathrm{m}$ thick buried oxide), and the $\mathrm{GeOI}$ root mean square surface roughness was as 

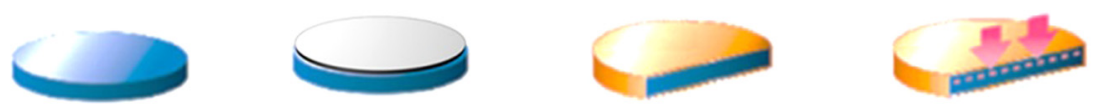

\section{$\begin{array}{lll}\text { (a) Substrate (b) Ge growth } & \text { (c) } \mathrm{SiO}_{2} \text { coating (d) Implantation }\end{array}$}

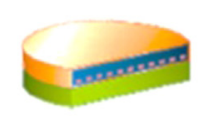

(e) Bonding

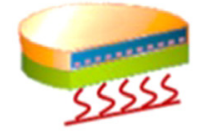

(f) Anneal

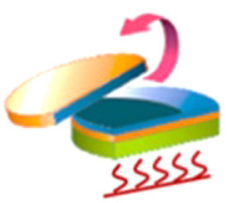

(g) Splitting

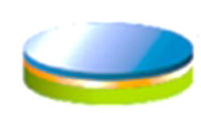

\section{(h) Finished}

low as $0.31 \mathrm{~nm}$. Finally, thinning is performed by dry etching in a RIE reactor in order to obtain the desirable Ge thickness for the targeted application. $0.35 \mu \mathrm{m}$ thickness was chosen for mono-mode light propagation at wavelengths close to $2 \mu \mathrm{m}$. This GeOI process flow yields higher crystalline quality layers compared to Ge directly grown on $\mathrm{Si}$ or SOI. Indeed, only the top part of thick Ge layers $(2.5 \mu \mathrm{m}$, typically), which is far less defective than the region close to the $\mathrm{Ge} / \mathrm{Si}$ interface, is present in the end on top of the BOX. At the end of the fabrication, the Ge strain is evaluated at $0.16 \%$. It results from differences in thermal expansion coefficients between $\mathrm{Ge}$ and $\mathrm{Si}$, which come into play during the cooling-down to room temperature after epitaxy (Fig. 1(b)). The strain is essentially preserved through the bonding steps (Fig. 1(e)-1(g)). This residual strain is later amplified via the lateral structuration of the suspended membrane.

High-Angle Annular Dark-Field imaging in a Transmission Electron Microscope (TEM) was used to compare SmartCut ${ }^{\mathrm{TM}}$ optical GeOI substrates and $1.4 \mu \mathrm{m}$ thick Ge layers grown on SOI substrates in the same CVD chamber. Cross-sectional micrographs can be found in Figure 2. For thick Ge layers grown on SOI (Figure 2(a)), numerous misfit dislocations are present close to the $\mathrm{Ge} / \mathrm{Si}$ interface, which is harmful for mechanical resistance to high strain. A close-up at the interface between Ge and the BOX is shown in Figure 2(b) for an optical GeOI substrate. Much lower number of dislocations is evidenced for the GeOI. X-ray diffraction (XRD) rocking curves were also measured in Ge grown on SOI and in GeOI substrates around the 220 Bragg reflection. The peak full width at half maximum was $30 \%$ smaller in $\mathrm{GeOI}$ substrates than in Ge-on-SOI, indicating an improvement in mosaicity for GeOI substrates (i.e., a better crystalline quality). In summary, at the end of the process, we obtained very high quality Ge-On-Insulator films with a built-in thermal strain.

The principle of the strain concentration ${ }^{2}$ is presented in Figure 3. The strain is concentrated in the central area of our device by suspending a patterned Ge layer with the typical profile presented in Figure 3(a). In order to optimize the strain homogeneity, we performed Finite Element Method (FEM) simulations using COMSOL Multiphysics. We used a 2D linear elastic model taking into account the germanium stiffness matrix. We assumed that there was no stress along the direction perpendicular to the membrane. An additional boundary condition is set as a zero displacement of the Ge layer along the contour defined by the under etching front (dashed line in the Figure 3(a)), and we apply a stress on the freed pattern (red line in the Figure 3(a)) which corresponds to the initial strain of $0.16 \%$ present in the layer. Figures 3(b)-3(d) show the computation of the biaxial strain $\left(\varepsilon_{\mathrm{xx}}+\varepsilon_{\mathrm{yy}}\right) / 2$ for membranes with different dimensions of the central zone. Dimensions a, b, d, e are, respectively, 250, 9, 17 , and $116 \mu \mathrm{m}$ with an under etching of $70 \mu \mathrm{m}$. The under etching value is the distance of the liberation presented by the parameters $u$ in the Figure 3(a). Only dimension $c$ is varying between Figures 1(b)-1(d). Keeping the under etching and the traction arm length identical, the strain increases when the size of the center zone decreases, giving a bi-axial tensile strains of $1.03,1.35$, and $1.90 \%$ for $\mathrm{c}=4,3$, and $2 \mu \mathrm{m}$, respectively. These calculated strains were averaged around $1 \mu \mathrm{m}$ in the central region (dashed black circle in the Figure 3).

To redistribute and amplify the tensile strain present in optical GeOI substrates, membranes were fabricated using the design rules presented in Figure 3. Membrane patterning (a)

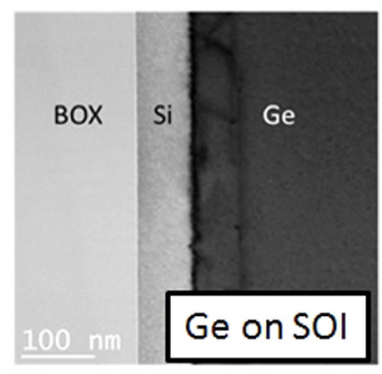

(b)

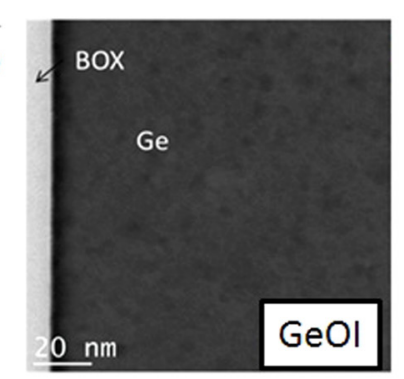

(c)

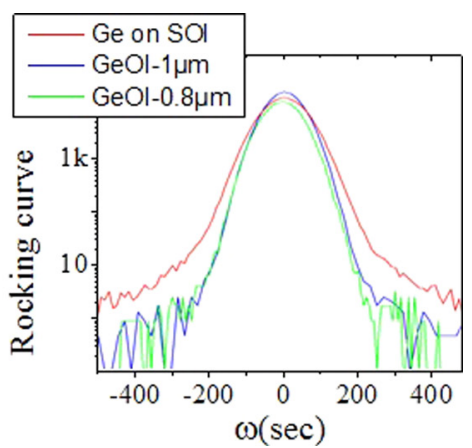

FIG. 2. Cross-sectional TEM images of (a) Ge grown on SOI and (b) GeOI showing a dramatic reduction of the density of misfit dislocations in GeOI; (c) XRD Rocking curves associated with GeOI and Ge grown on thinned SOI. 
(a)

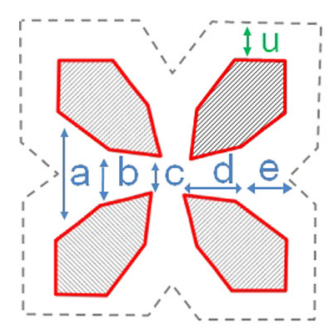

(b)

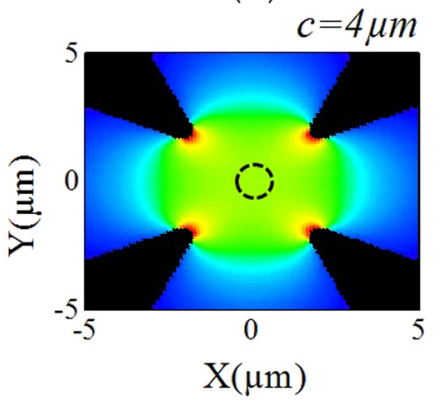

(c)

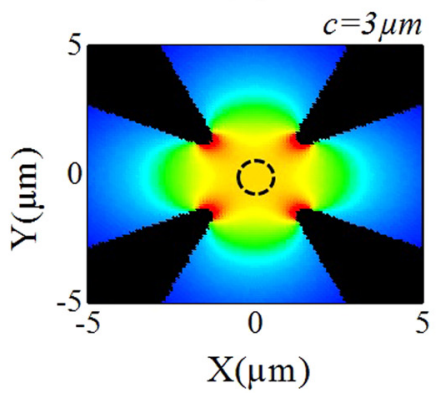

(d)

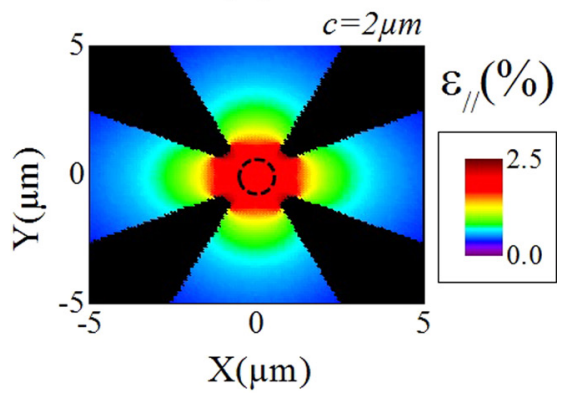

FIG. 3. (a) Schematics representation of the etched regions in the germanium membrane showing the various parameters used to optimize strain in the central region; Finite Element Method simulation of a bi-axially stressed membrane for the dimensions indicated in the figure with an under etching of $70 \mu \mathrm{m}$ for (b) $\mathrm{c}=3.7 \mu \mathrm{m} ;(\mathrm{c}) \mathrm{c}=2.7 \mu \mathrm{m} ;$ and $(\mathrm{d}) \mathrm{c}=2 \mu \mathrm{m}$.

was performed using e-beam lithography followed by dry etching in an ICP reactor with $\mathrm{Cl}_{2}, \mathrm{~N}_{2}$, and $\mathrm{O}_{2}$ gas. The under-etching was carried out in a dedicated etching reactor combining anhydrous HF vapor and alcohol vapors. Etching speed could be modified by varying the gas flows. Low speed under-etching yielded Ge membranes suspended in the air, as illustrated by SEM imaging in Figure 4(b).

Tensile strain was quantified using micro-Raman spectroscopy with an input laser at $785 \mathrm{~nm}$ wavelength (giving a light penetration depth of $220 \mathrm{~nm}$ ). The light was focused onto the sample surface with a $100 \times(0.9$ numerical aperture $)$ short working distance objective. The resulting spot diameter was around $1 \mu \mathrm{m}$. Power dependence measurements were performed in order to quantify the heating effect. ${ }^{17}$ From this calibration, the laser intensity was fixed to $11 \mu \mathrm{W}$ focused on the sample which gives a heating effect lower than the indicated uncertainty of $0.1 \mathrm{~cm}^{-1}$. The Raman shift is measured by fitting spectra with Lorentzian functions in order to increase accuracy. A bulk Ge (001) substrate was systematically used as a reference for $0 \%$ of deformation. The strain shift coefficient used to linearly link the applied strain and the detected Raman shift was $424 \mathrm{~cm}^{-1} \cdot{ }^{18-20}$ Figure 5(a) presents the Raman spectra associated with an optical GeOI wafer and a bulk Ge (001) substrate. The tensile strain in the former is around $0.16 \%$. The Raman spot size is well suited to measure the strain in the center of Ge membranes given the strain homogeneity presented in Figure 3 (as illustrated in the inset of Figure 5(b)). Parameters a, b, d, and e presented in Figure 1 are 250, 9, 17, and $116 \mu \mathrm{m}$, respectively. The under-etching is evaluated to

(a)

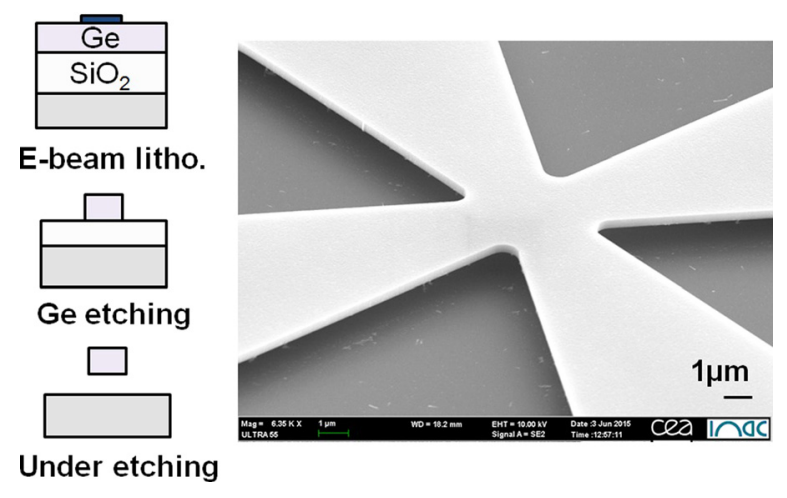

FIG. 4. (a) Schematics representation of the process flow used for membrane fabrication and (b) inclined SEM imaging of a studied Ge membrane. be around $70 \mu \mathrm{m}$ by optical microscopy measurements. When the dimension $\mathrm{c}$ of the strained regions shrinks (for the same traction arms) from 4 down to 3 and finally $2 \mu \mathrm{m}$, the measured tensile strain increases from $1.1 \%$ up to $1.4 \%$ and finally a record breaking $1.9 \%$. Such data are in line with values predicted by modelling (Fig. 3). The maximum detected Raman spectral shift is $8.1 \pm 0.1 \mathrm{~cm}^{-1}$, which corresponds to a strain value of $1.91 \pm 0.02 \%$.

(a)

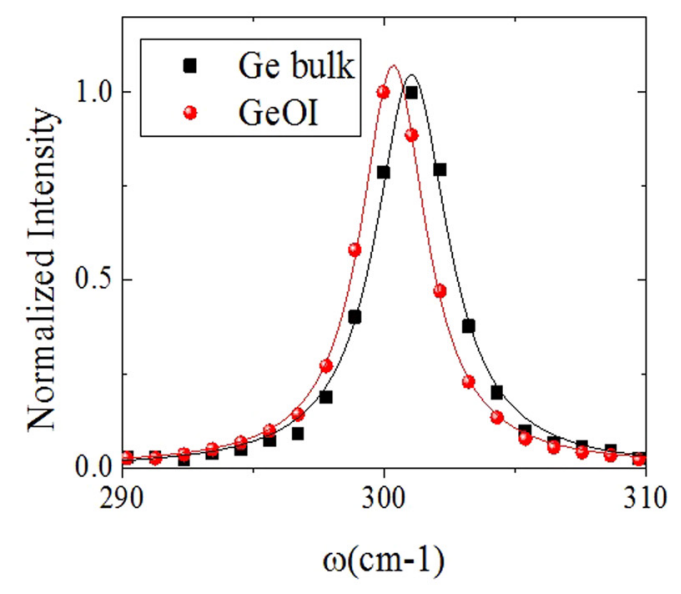

(b)

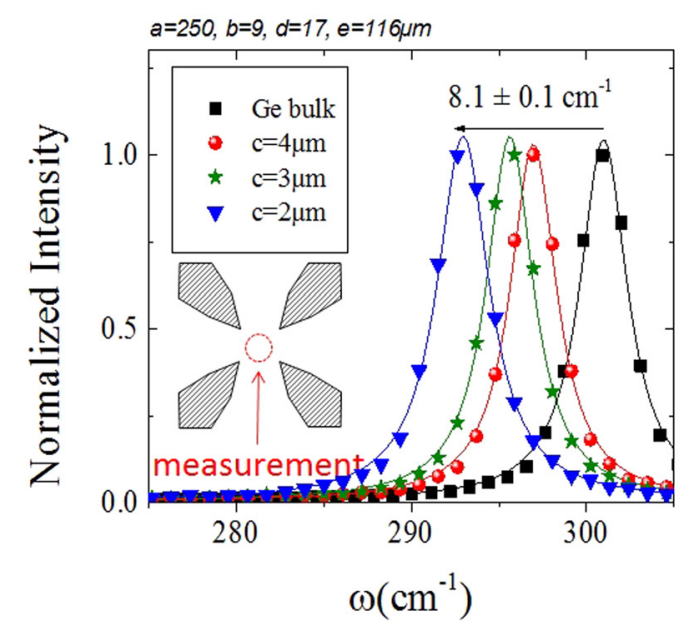

FIG. 5. Raman spectroscopy measurement: (a) for Ge bulk substrate and blanket optical GeOI substrate; and (b) for different Ge membranes measured in the center and a Ge bulk substrate. 
Highly strained Ge membranes were fabricated using biaxial strain amplification in optical GeOI substrates. Thanks to the high quality of SmartCut ${ }^{\mathrm{TM}} \mathrm{GeOI} 200 \mathrm{~mm}$ wafer, tensile strains up to $1.9 \%$ in thick Ge layers $(350 \mathrm{~nm})$ were achieved. We have thus reached the strain value predicted to transform germanium into a direct band-gap material. This work highlights the $200 \mathrm{~mm}$ GeOI wafer as a very promising platform for Ge photonics. Indeed, such high induced strain in thick Ge layer shows the high potential of tensile strain redistribution in GeOI wafer and paves the way toward the demonstration of a Ge direct bandgap for laser applications.

The authors would like to thank Patrice Gergaud and the Platforme de Technologie Amont in Grenoble for XRD and clean room facilities. This work was supported by the CEA DSM-DRT Phare Projects Photonics and Operando.

${ }^{1}$ J. Liu, X. Sun, D. Pan, X. Wang, L. C. Kimerling, T. L. Koch, and J. Michel, "Tensile-strained, n-type Ge as a gain medium for monolithic laser integration on Si," Opt. Express 15(18), 11272-11277 (2007).

${ }^{2}$ M. J. Suess, R. Geiger, A. R. Minamisawa, G. Schiefler, J. Frigerio, D. Chrastina, G. Isella, R. Spolenak, J. Faist, and H. Sigg, "Analysis of enhanced light emission from highly strained germanium microbridges," Nat. Photonics 7(6), 466-472 (2013).

${ }^{3}$ D. S. Sukhdeo, D. Nam, J.-H. Kang, M. L. Brongersma, and K. C. Saraswat, "Direct bandgap germanium-on-silicon inferred from 5.7\% $\langle 100\rangle$ uniaxial tensile strain," Photonics Res. 2, A8 (2014).

${ }^{4}$ S. Wirths, R. Geiger, N. von den Driesch, G. Mussler, T. Stoica, S. Mantl, Z. Ikonic, M. Luysberg, S. Chiussi, J. M. Hartmann, H. Sigg, J. Faist, D. Buca, and D. Grützmacher, "Lasing in direct-bandgap GeSn alloy grown on Si," Nat. Photonics 9(2), 88-92 (2015).

${ }^{5}$ C. G. de Walle, "Band lineups and deformation potentials in the modelsolid theory," Phys. Rev. B 39(3), 1871-1883 (1989).

${ }^{6}$ D. S. Sukhdeo, D. Nam, J.-H. Kang, M. L. Brongersma, and K. C. Saraswat, "Bandgap-customizable germanium using lithographically determined biaxial tensile strain for silicon-compatible optoelectronics," Opt. Express 23(13), 16740 (2015).

${ }^{7}$ H. Tahini, A. Chroneos, R. W. Grimes, U. Schwingenschloegl, and A. Dimoulas, "Strain-induced changes to the electronic structure of germanium," J. Phys.: Condens. Matter 24(19), 195802 (2012).

${ }^{8}$ M. El Kurdi, G. Fishman, S. Sauvage, and P. Boucaud, "Band structure and optical gain of tensile-strained germanium based on a 30 band $\mathrm{kp}$ formalism,” J. Appl. Phys. 107, 013710 (2010).
${ }^{9}$ J. R. Sanchez-Perez, C. Boztug, F. Chen, F. F. Sudradjat, D. M. Paskiewicz, R. B. Jacobson, M. G. Lagally, and R. Paiella, "Directbandgap light-emitting germanium in tensilely strained nanomembranes," Proc. Natl. Acad. Sci. U. S. A. 108(47), 18893-18898 (2011).

${ }^{10}$ Y. Huo, H. Lin, R. Chen, M. Makarova, Y. Rong, M. Li, T. I. Kamins, J. Vuckovic, and J. S. Harris, "Strong enhancement of direct transition photoluminescence with highly tensile-strained Ge grown by molecular beam epitaxy," Appl. Phys. Lett. 98, 011111 (2011).

${ }^{11}$ K. Guilloy, N. Pauc, A. Gassenq, P. Gentile, S. Tardif, F. Rieutord, and V. Calvo, "Tensile strained germanium nanowires measured by photocurrent spectroscopy and X-ray microdiffraction," Nano Lett. 15(4), 2429-2433 (2015).

${ }^{12}$ A. Ghrib, M. El Kurdi, M. Prost, S. Sauvage, X. Checoury, G. Beaudoin, M. Chaigneau, R. Ossikovsk, I. Sagnes, and P. Boucaud, "All-around SiN stressor for high and homogeneous tensile strain in germanium microdisk cavities," Adv. Opt. Mater. 3(3), 353-358 (2015).

${ }^{13}$ J. Petykiewicz, D. Nam, D. S. Sukhdeo, S. Gupta, S. Buckley, A. Y. Piggott, J. Vučković, and K. C. Saraswat, "Direct bandgap light emission from strained Ge nanowires coupled with high-Q optical cavities," e-print arXiv:1508.01255.

${ }^{14}$ C. Deguet, L. Sanchez, T. Akatsu, F. Allibert, J. Dechamp, F. Madeira, F. Mazen, A. Tauzin, V. Loup, C. Richtarch, D. Mercier, T. Signamarcheix, F. Letertre, B. Depuydt, and N. Kernevez, "Fabrication and characterisation of $200 \mathrm{~mm}$ germanium-on-insulator (GeOI) substrates made from bulk germanium," Electron. Lett. 42, 415 (2006).

${ }^{15}$ F. Letertre, C. Deguet, C. Richtarch, B. Faure, J. Hartmann, F. Chieu, A. Beaumont, J. Dechamp, C. Morales, F. Allibert, P. Perreau, S. Pocas, S. Personnic, C. Lagahe-Blanchard, B. Ghyselen, Y. Le Vaillant, Jalaguier, N. Kernevez, and C. Mazure, "Germanium-On-Insulator (GeOI) structure realized by the Smart Cut ${ }^{\mathrm{TM}}$ technology," MRS Proc. 809, B4.4 (2004).

${ }^{16}$ V. Reboud, J. Widiez, J. Michel, G. Osvaldo, D. Fowler, A. Chelnokov, A. Gassenq, K. Guilloy, N. Pauc, V. Calvo, R. Geiger, T. Zabel, J. Faist, and H. Sigg, "Structural and optical properties of $200 \mathrm{~mm}$ germanium-on- insulator (GeOI) substrates for silicon photonics applications," Proc. SPIE 936714, 1-6 (2015).

${ }^{17}$ M. Süess, R. Minamisawa, R. Geiger, K. Bourdelle, H. Sigg, and R. Spolenak, "Power-dependent Raman analysis of highly strained Si nanobridges," Nano Lett. 14(3), 1249-1254 (2014).

${ }^{18}$ I. De Wolf, "Micro-Raman spectroscopy to study local mechanical stress in silicon integrated circuits," Semicond. Sci. Technol. 11, 139 (1996).

${ }^{19}$ F. Cerdeira, C. J. Buchenauer, F. H. Pollak, and M. Cardona, "Stressinduced Raman shifts of diamond and zincblende semicond," Phys. Rev. B 5(2), 580 (1972).

${ }^{20}$ F. Pezzoli, E. Grilli, M. Guzzi, S. Sanguinetti, D. Chrastina, G. Isella, H. von Känel, E. Wintersberger, J. Stangl, and G. Bauer, "Strain-induced shift of phonon modes in alloys," Mater. Sci. Semicond. Process. 9(4-5), $541-545$ (2006). 